\title{
Excellent Stability in Water of Single-Chain Nanoparticles against Chain Scission by Sonication ${ }^{\mathrm{a}}$
}

Marina González-Burgos, Edurne González, and José A. Pomposo*

M. Gonzalez-Burgos, Dr. E. Gonzalez, Prof. J. A. Pomposo

Centro de Física de Materiales (CSIC, UPV/EHU) - Materials Physics Center MPC

Paseo Manuel de Lardizábal 5, 20018 San Sebastián, Spain

E-mail: Josetxo.pomposo@ehu.eus

M. Gonzalez-Burgos, Prof. J. A. Pomposo

Departamento de Física de Materiales

Universidad del País Vasco (UPV/EHU)

Apartado 1072, 20800 San Sebastián, Spain

Prof. J. A. Pomposo

IKERBASQUE - Basque Foundation for Science

Maria Díaz de Haro 3, 48013 Bilbao, Spain

We report herein on the superior stability against degradation induced by ultrasound irradiation of water-soluble single-chain polymer nanoparticles when compared to their parent precursor polymers, and we suggest a loop scission mechanism in support of such behavior.

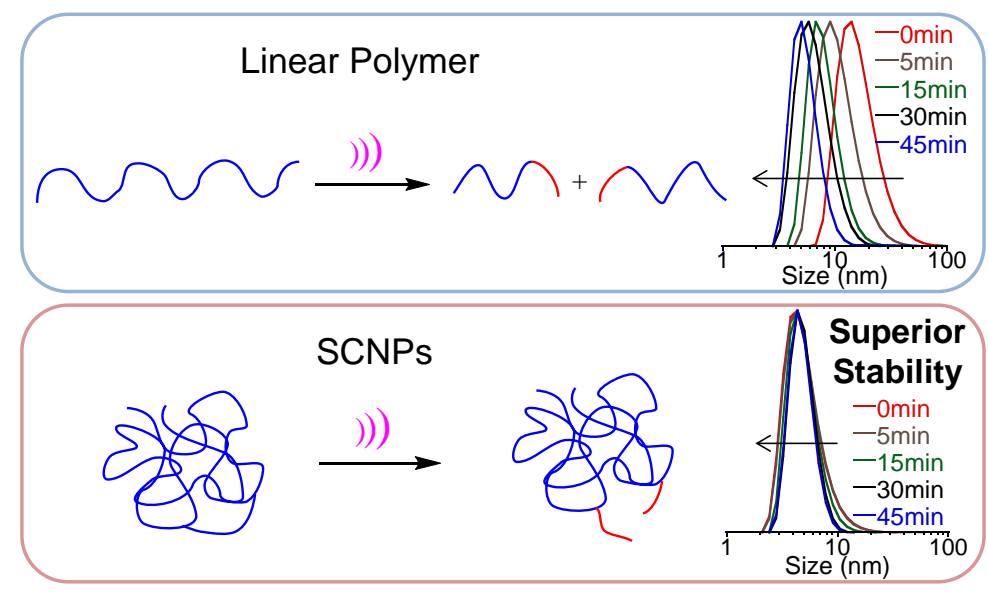

\footnotetext{
${ }^{\text {a }}$ Supporting Information is available online
} 


\section{Introduction}

Inspired by the folding of natural macromolecules to functional bio-entities, the compaction of individual polymer chains to single-chain nanoparticles (SCNPs) is a field that has attracted significant interest in recent years. ${ }^{[1]}$ The folding of isolated non-natural chains can be a dynamic or irreversible process induced by reversible (i.e., non-covalent and dynamic covalent) interactions or covalent bonds, respectively. ${ }^{[2-4]}$ Examples of dynamic interactions utilized in the synthesis of responsive SCNPs are hydrogen bonds, ${ }^{[5]}$ metal-ligand complexation $^{[6]}$ and dynamic enamine covalent bonds. ${ }^{[7]}$ Such nanoparticles can be disassembled to their parent precursor polymers by means of one or several appropriate stimuli (e.g., temperature, $\mathrm{pH}$, competitive host compounds). ${ }^{[8]}$ The resulting SCNPs are reversible, ultra-fine soft nano-objects $(<20 \mathrm{~nm}$ ) showing promising properties for their use in nanomedicine and biomimetic catalysis, among other potential applications. ${ }^{[9]}$ When compared to these active soft nano-objects, SCNPs constructed by means of intramolecular covalent bonds have permanent intra-chain cross-links that affect to a large extent the final compaction degree achieved and often endow them with increased stability against thermal degradation at high temperatures. ${ }^{[10]} \mathrm{A}$ variety of highly-efficient cross-linking chemistries have been applied to synthesize covalent-bonded SCNPs such as the copper-catalysed azidealkyne click chemistry, Glaser-Hay coupling, thiol-ene/thiol-yne coupling reactions, etc. ${ }^{[1]}$ Potential applications of these SCNPs include their use as additives for conventional thermoplastic materials to produce so-called all-polymer nanocomposites, ${ }^{[12]}$ and the development of stabilized supported catalysts, especially if the reactions are performed at relatively high temperature. ${ }^{[13]}$

In addition to thermal stability, several potential applications of covalent-bonded SCNPs in nanomedicine do depend critically on their mechanical stability (e.g., artificial synoidal fluids for joint lubrication). In fact, mechanical force has been demonstrated to promote several chemical reactions, with sonication experiments illustrating the degradation 
of a variety of natural macromolecules (cellulose, polypeptides, polysaccharides, proteins, DNA) and synthetic polymers via force-induced bond rupture. ${ }^{[14]}$ When ultrasound is used to apply force to dilute polymer solutions, polymer chain scission is observed as a consequence of solvodynamic shear caused by cavitation: the nucleation, growth and collapse of bubbles in solution. It is generally accepted that the solvodynamic shear elongates the polymer backbone leading to scission that generally occurs near the midpoint of the polymer chain, where solvodynamic forces are the greatest. ${ }^{[15]}$ Consequently, a progressive reduction in the molecular weight (and polymer size) and a certain decrease in chain length polydispersity are often observed during sonication experiments of dilute polymer solutions. ${ }^{[14]}$ The absence of data about the mechanical stability of SCNPs under ultrasound irradiation in water led us to carry out sonication experiments for water-soluble covalent-bonded SCNPs and their corresponding precursor polymers under exactly the same conditions. ${ }^{[16]}$ Herein, we report on the superior stability against degradation induced by ultrasound irradiation of water-soluble covalent-bonded SCNPs when compared to their parent precursor polymers, as determined by a combination of size exclusion chromatography (SEC) and dynamic light scattering (DLS) measurements.

\section{Results and Discussion}

\subsection{Synthesis of Water-Soluble Poly( $N$-Vinyl Pyrrolidone) Single-Chain Nanoparticles}

As water-soluble single-chain nanoparticles, we prepared poly( $N$-vinyl pyrrolidone) (PVP) SCNPs (denoted as N1) starting from a linear PVP of high molecular weight (360 kDa) (P1) via Fenton reaction-promoted intramolecular radical coupling at high dilution (for experimental details: see Scheme 1, and Supporting Information, SI). PVP is a valuable water-soluble polymer with a large commercial use in medicine.

Confirmation of the successful formation of PVP SCNPs was obtained by combined SEC and DLS measurements, as well as ${ }^{1} \mathrm{H}$ nuclear magnetic resonance (NMR) and Fourier 
transform infrared (FTIR) spectroscopy results. A clear reduction of hydrodynamic size upon intra-chain coupling of radicals generated by Fenton reaction was observed in both SEC and DLS experiments (see SI). The resulting PVP SCNPs were soluble in water and no sign of the presence of multi-chain aggregates upon SCNP formation was observed by SEC or DLS. Complementary, changes which can be attributed to coupling of pyrrolidone moieties were observed in both ${ }^{1} \mathrm{H}$ NMR and FTIR spectra (see Figure 1). Hence, as a consequence of intramolecular pyrrolidone dimerization a split and shift of the carbonyl IR vibration band towards lower wavenumbers was observed (see inset in Figure 1a). Moreover, the appearance of a new broad peak at $2.6 \mathrm{ppm}$ in the ${ }^{1} \mathrm{H}$ NMR spectrum of the PVP SCNPs was observed that we assign to $-\mathrm{CH}$ - protons from intra-chain pyrrolidone dimers (dashed zone in Figure 1b). Based on the area of this new peak, we estimated a value of intra-chain cross-linking of ca. 10 mol \% (i.e., on average 1 every 10 pyrrolidone units was intramolecularly crosslinked).

\subsection{Ultrasound Irradiation of Linear Precursors and Water-Soluble Single-Chain}

\section{Nanoparticles}

As a control experiment, we initially subjected the precursor polymer P1 to ultrasound irradiation in water and monitored the evolution of its SEC trace upon ultrasound irradiation as a function of time (see Figure 2). In SEC experiments, macromolecules are separated according to their respective hydrodynamic size and consequently molecular weight, where bigger macromolecules show shorter SEC retention time than smaller ones. ${ }^{[17]}$ As illustrated in Figure 2a the SEC trace of $\mathbf{P 1}$ is found to shift progressively towards longer retention times (lower molecular weight / smaller size) upon increasing sonication time. This fact can be attributed to degradation by chain scission which reduces progressively the length of the PVP chains. The results are in good agreement with prior sonication experiments involving PVP. ${ }^{[18]}$ To our surprise, however, the SEC trace of N1 (Figure 2b) showed only a very minor 
shift even after 45 min of sonication, pointing to an excellent stability against degradation induced by ultrasound irradiation of the water-soluble single-chain nanoparticles when compared to the linear precursor polymer. The effect of sonication time on the molecular weight at the peak maximum $\left(M_{\mathrm{app}}\right)$ for $\mathbf{P 1}$ and $\mathbf{N 1}$ is displayed in Figure 2c. Results from triplicate experiments were found to be highly reproducible (Table S2). Also, a similar trend was observed when the values of number average molecular weight $\left(M_{\mathrm{n}}\right)$ or weight average molecular weight $\left(M_{\mathrm{w}}\right)$ were used instead of $M_{\mathrm{app}}$ (Table S3). Concerning P1, even if the sonication-induced chain scission process presumably introduces a large number of chain-end groups, we were not able to quantify them by conventional techniques like FTIR or ${ }^{1} \mathrm{H}$ NMR spectroscopy.

The above intriguing SEC results were corroborated by DLS measurements in which the average hydrodynamic size $\left(R_{\mathrm{h}}\right)$ of the SCNPs and the parent polymer precursors, as well as their complete $R_{\mathrm{h}}$ distributions were determined as a function of ultrasound irradiation time (see Figure 3). A progressive reduction in the value of $R_{\mathrm{h}}$ as a function of sonication time was observed for P1 (Figure 3a), whereas N1 showed no significant change even after 45 min of ultrasound irradiation time (Figure 3b) in good agreement with the previous SEC results. In fact, after 45 min of solvodynamic stress the size of $\mathbf{P 1}$ reduces from 17.2 to $5.6 \mathrm{~nm}$, while the size of N1 only from 5.2 to $4.8 \mathrm{~nm}$, which is within the experimental error of the DLS technique (Figure S2). Taken together, the SEC and DLS results illustrate the excellent mechanical stability in water of single-chain nanoparticles against chain scission by ultrasound irradiation.

\subsection{Loop Scission Mechanism}

Concerning the mechanism of improved mechanical stability of SCNPs when compared to linear polymers, it is generally accepted that because chain scission is a nonrandom process (with cleavage near the chain center), it is not a thermal process. ${ }^{[19]}$ Figure 3c shows a 
comparison of the thermal stability of $\mathbf{P 1}$ and $\mathbf{N 1}$ as recorded by thermogravimetic analysis (TGA) measurements. P1 and N1 (which are highly hygroscopic materials) displayed approximately the same thermal stability up to $200{ }^{\circ} \mathrm{C}$, but $\mathbf{N 1}$ showed a reduced thermal stability above this temperature. Consequently, the improved mechanical stability of N1 when compared to $\mathbf{P 1}$ seems to be unrelated to a thermally activated process.

Instead of the accepted chain scission mechanism operative in linear polymers ${ }^{[14,15]}$ (see Scheme 2a), we suggest here a different mechanism involving local loop scission events that accounts for the superior stability exhibited by single-chain polymer nanoparticles against degradation by ultrasound irradiation (Scheme 2b). Hence, instead of a reduction in molecular weight / polymer size upon force-induced bond rupture as observed for linear polymers, the successive loop scission events taken place in SCNPs under ultrasound irradiation will affect only at a smaller local scale without giving to global chain cleavage. In particular, the proposed loop scission mechanism is supported by: i) the nearly constant molecular weight of the single-chain nanoparticles even at large sonication times (Figure 2b,c) since force-induced bond rupture produce fragments that remain bonded to the SCNP; ii) the nearly constant hydrodynamic size of the single-chain nanoparticles even at high ultrasound irradiation times (Figure 3c,d and Figure S2) since no significant change in global size is expected upon a few local loop scission events (by the contrary, midpoint chain fragmentation reduces significantly the hydrodynamic size due to acute shortening of the chain); and iii) no thermal activated processes need to be invoked to support the excellent stability against degradation induced by ultrasound irradiation of water-soluble SCNPs when compared to their parent linear precursor polymers.

Obviously, the loop scission rate will depend on the specific chemical composition of the SCNPs since -at the end- scission involves force-induced bond rupture that depends on the strength of the bond. ${ }^{[20]}$ 


\section{Conclusions}

We report on the excellent stability against degradation induced by ultrasound irradiation of water-soluble covalent-bonded SCNPs when compared to their parent linear precursor polymers, as determined by a combination of SEC and DLS measurements. Instead of the accepted chain scission mechanism operative in linear polymers, we suggest a different mechanism for SCNPs under sonication involving local loop scission events that accounts for the observed experimental behavior. Further works including computational studies are needed to validate the loop scission mechanism and to know the effect of molecular weight, cross-linking degree, type of cross-linking chemistry, etc. on SCNP stability under sonication. Anyway, the increased stability of SCNPs against solvodynamic shear offers new opportunities for the development of a new generation of mechanically stable ultra-fine soft nanomaterials.

\section{Supporting Information}

Supporting Information is available from the Wiley Online Library or from the author.

Acknowledgements: Financial support by the Spanish Ministry "Ministerio de Economia y Competitividad", MAT2015-63704-P (MINECO / FEDER, UE), the Basque Government, IT654-13, and the Gipuzkoako Foru Aldundia, RED 101/17, is acknowledged. M. G.-B. is grateful to the University of the Basque Country for her UPV/EHU pre-doctoral grant. E. G. received funding from the "Fellows Gipuzkoa” fellowship of the Gipuzkoako Foru Aldundia.

Received: Month XX, XXXX; Revised: Month XX, XXXX; Published online: Month XX, XXXX

DOI: 10.1002/marc.2017\#\#\#\#\#

Keywords: Nanoparticles, Sonication, Chain scission, Ultrasound, Stability 
[1] Single-Chain Polymer Nanoparticles: Synthesis, Characterization, Simulations and Applications, Ed.: J. A. Pomposo, Wiley-VCH, Weinheim, Germany, 2017.

[2] O. Altintas, C. Barner-Kowollik, Macromol. Rapid Commun. 2016, 37, 29.

[3] M. Gonzalez-Burgos, A. Latorre-Sanchez, J. A. Pomposo, Chem. Soc. Rev. 2015, 44, 6122.

[4] C. K. Lyon, A. Prasher, A. M. Hanlon, B. T. Tuten, C. A. Tooley, P. G. Frank, E. B. Berda, Polym. Chem. 2015, 6, 181.

[5] a) T. Terashima, T. Mes, T. F. A. De Greef, M. A. J. Gillissen, P. Besenius, A. R. A. Palmans, E. W. Meijer, J. Am. Chem. Soc. 2011, 133, 4742.; b) E. J. Foster, E. B. Berda, E. W. Meijer, J. Am. Chem. Soc. 2009, 131, 6964; c) M. Seo, B. J. Beck, J. M. J. Paulusse, C. J. Hawker, S. Y. Kim, Macromolecules 2008, 41, 6413.

[6] a) J. Willenbacher, O. Altintas, V. Trouillet, N. Knöfel, M. J. Monteiro, P. W. Roesky, C. Barner-Kowollik, Polym. Chem. 2015, 6, 4358; b) A. Sanchez-Sanchez, A. Arbe, J. Colmenero, J. A. Pomposo, ACS Macro Lett. 2014, 3, 439; c) S. Mavila, C. E. Diesendruck, S. Linde, L. Amir, R. Shikler, N. G. Lemcoff, Angew. Chem., Int. Ed. 2013, 52, 5767.

[7] A. Sanchez-Sanchez, D. A. Fulton, J. A. Pomposo, Chem. Commun. 2014, 50, 1871.

[8] T. S. Fischer, D. Schulze-Sünninghausen, B. Luy, O. Altintas, C. Barner-Kowollik, Angew. Chem. Int. Ed. 2016, 55, 11276.

[9] a) A. Latorre-Sánchez, J. A. Pomposo, Polym. Int. 2016, 65, 855; b) M. Huo, N. Wang, T. Fang, M. Sun, Y. Wei, J. Yuan, Polymer 2015, 66, A11.

[10] J. De-La-Cuesta, E. González, A. J. Moreno, A. Arbe, J. Colmenero, J. A. Pomposo, Macromolecules 2017, 50, 6323.

[11] S. Mavila, O. Eivgi, I. Berkovich, N. G. Lemcoff, Chem. Rev. 2016, 116, 878.

[12] M. E. Mackay, T. T. Dao, A. Tuteja, D. L. Ho, B. V. Horn, H.-C. Kim, C. J. Hawker, Nature Mater. 2003, 2, 762.

[13] a) N. D. Knöfel, H. Rothfuss, J. Willenbacher, C. Barner-Kowollik, P. W. Roesky, Angew. Chem. Int. Ed. 2017, 56, 4950; b) J. Rubio-Cervilla, E. González, José A. Pomposo. Nanomaterials 2017, 7, 341.

[14] M. M. Caruso, D. A. Davis, Q. Shen, S. A. Odom, N. R. Sottos, S. R. White, J. S. Moore, Chem. Rev. 2009, 109, 5755.

[15] K. S. Suslick, G. J. Price, Annu. Rev. Mater. Sci. 1999, 29, 295.

[16] During the preparation of the present communication a preliminary work has been published about the mechanochemistry of intramolecular cross-linked polymers under 
ultrasound irradiation in organic media: A. Levy, F. Wang, A. Lang, O. Galant, C. E. Diesendruck, Angew. Chem. Int. Ed. 2017, 56, 6431.

[17] Light Scattering, Size Exclusion Chromatography and Asymmetric Flow Field Flow Fractionation. S. Podzimek, John Wiley \& Sons, Inc., Hoboken, NJ, 2011.

[18] S. Koda, H. Mori, K. Matsumoto, H. Nomura, Polymer 1994, 35, 30.

[19] B. M. E. van der Hoff, C. E. Gall, J. Macromol. Sci. 1997, A11, 1739.

[20] M. V. Encina, E. Lissi, M. Sarasua, L. Gallo, D. Radic, J. Polym. Sci.; Polym. Phys. Ed. 1980, 18, 757. 


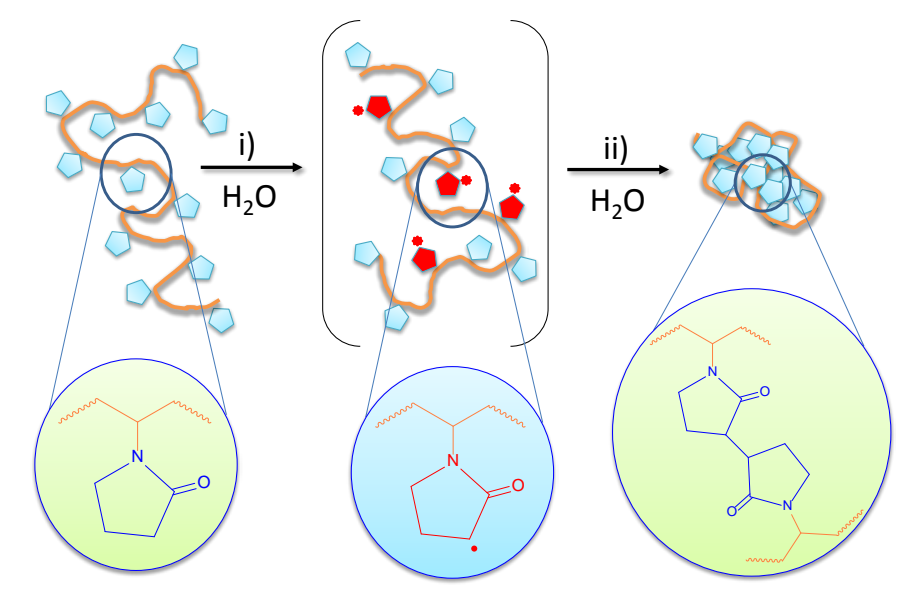

Scheme 1. Illustration of the synthesis at high dilution of water-soluble poly(vinyl pyrrolidone) single-chain nanoparticles (PVP SCNPs) via intra-chain coupling (step ii) of radicals generated by Fenton reaction (step i) (see SI).
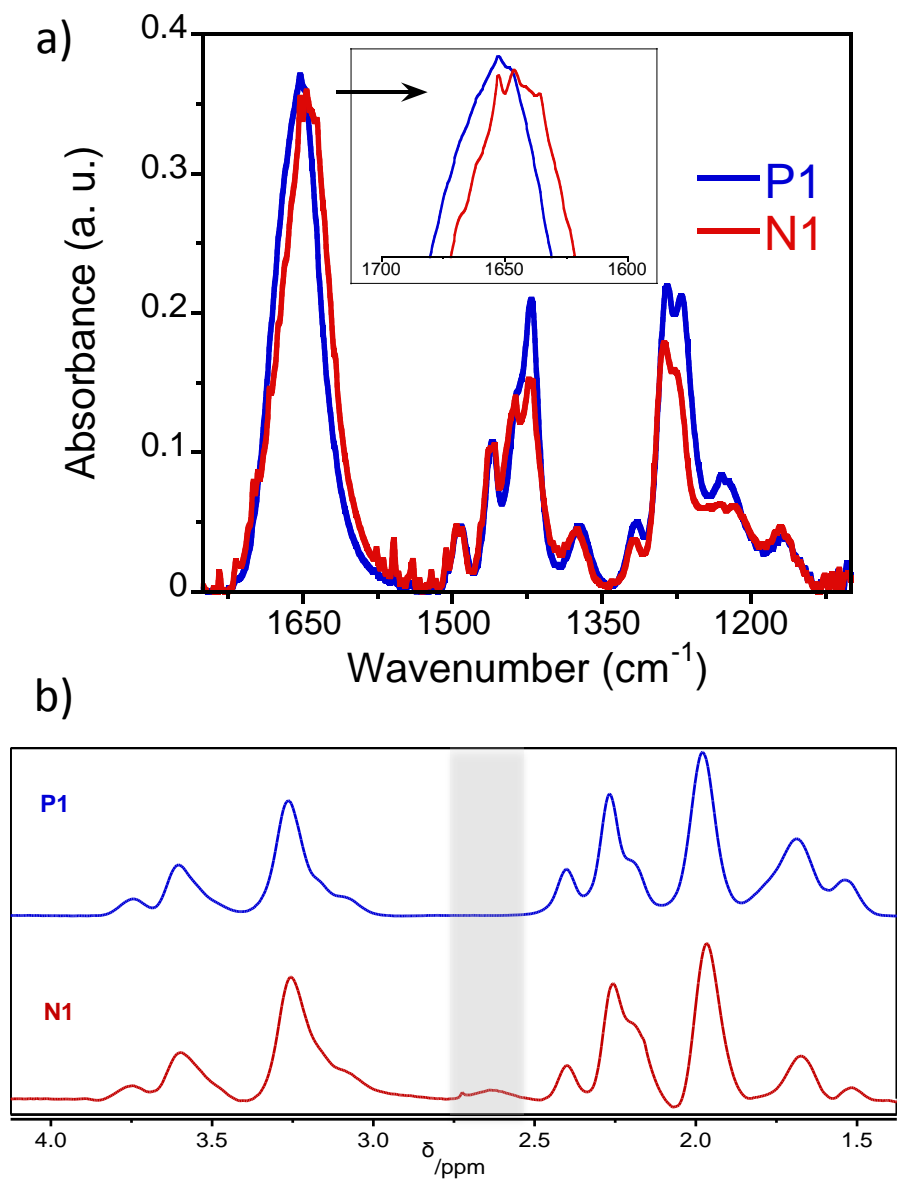

Figure 1. Comparison of FTIR (a) and ${ }^{1} \mathrm{H}$ NMR (b) spectra of $\mathbf{P 1}$ and N1. Changes observed upon SCNP formation are highlighted by the arrow in (a) and the dashed zone in (b), respectively. 

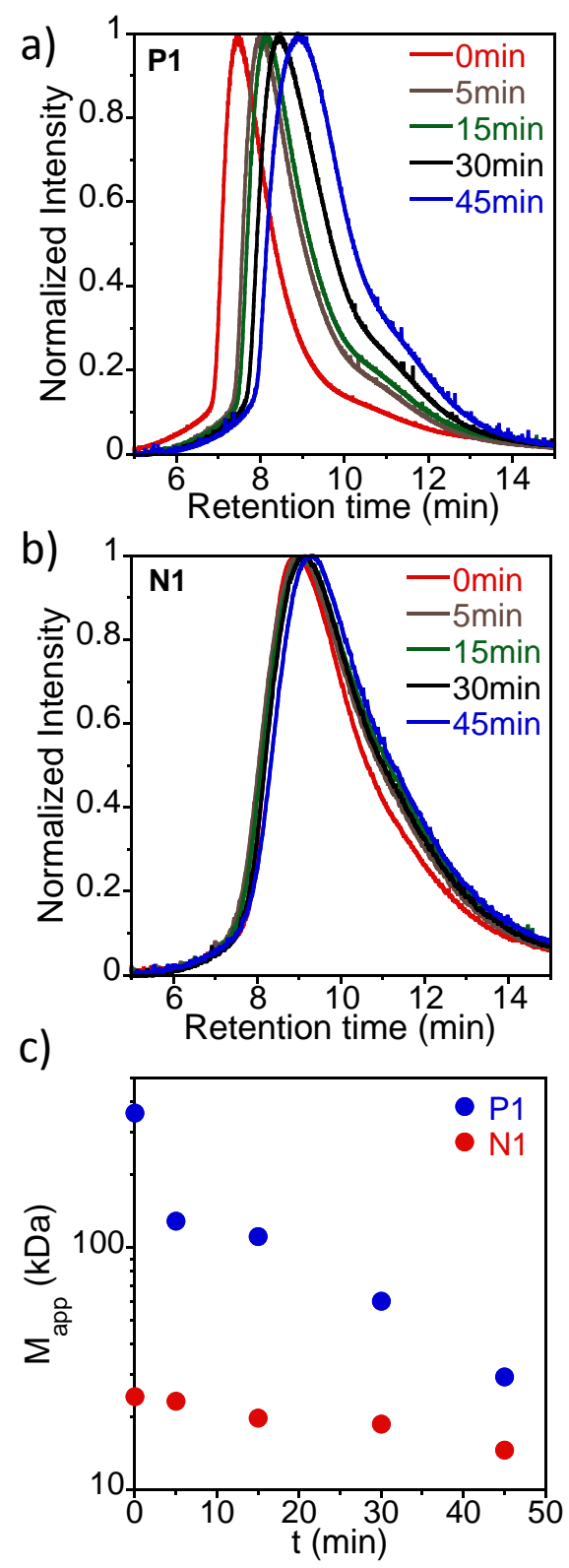

Figure 2. Evolution of the size exclusion chromatography (SEC) traces of $\mathbf{P 1}$ (a) and N1 (b) with ultrasound irradiation time, and effect of sonication time on the molecular weight at the peak maximum ( $M_{\text {app }}$ ) of P1 and N1 (c). 

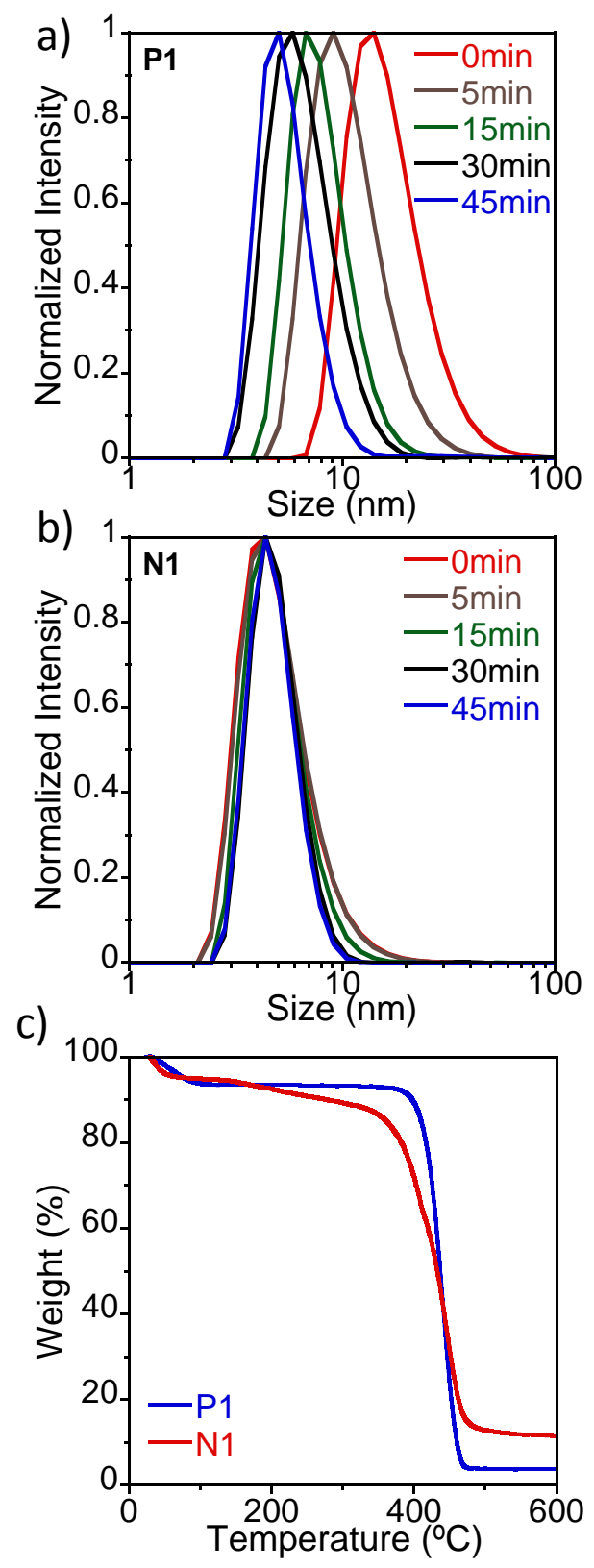

Figure 3. Evolution of the distribution of hydrodynamic size of $\mathbf{P 1}$ (a) and N1 (b) with ultrasound irradiation time as determined by dynamic light scattering (DLS) experiments, and comparison of thermal stability of $\mathbf{P 1}$ and $\mathbf{N 1}$ (c) as recorded by thermogravimetic analysis (TGA) measurements. 
a)

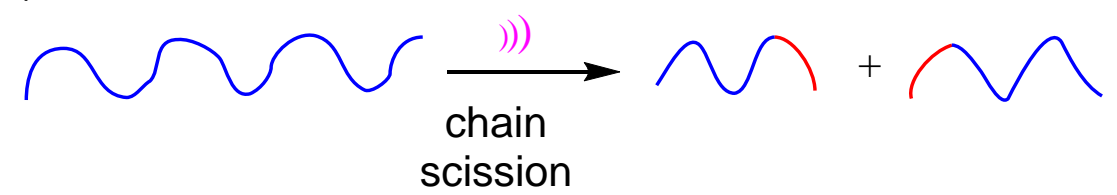

b)
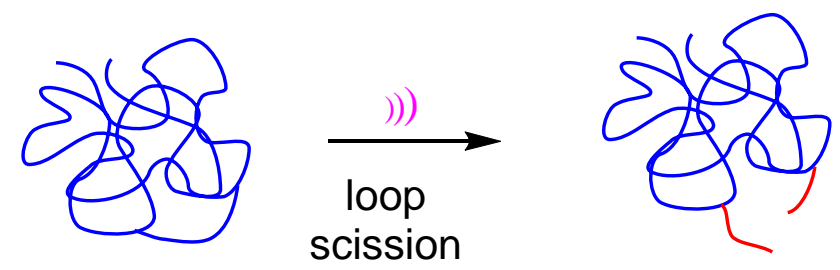

Scheme 2. Cartoon representation of sonication-induced chain scission mechanism operative in linear polymers (a), and proposed mechanism to support the superior stability of singlechain polymer nanoparticles against degradation by ultrasound irradiation, involving loop scission events (b). In both cases, the fragments involved in force-induced bond rupture are shown in red colour. 